\title{
Human Milk Oligosaccharides: Evolution, Structures and Bioselectivity as Substrates for Intestinal Bacteria
}

\author{
J. Bruce German, Samara L. Freeman, \\ Carlito B. Lebrilla, David A. Mills
}

Department of Nutrition, University of California, Davis, CA, USA

\begin{abstract}
Human milk contains a high concentration of diverse soluble oligosaccharides, carbohydrate polymers formed from a small number of monosaccharides. Novel methods combining liquid chromatography with high resolution mass spectrometry have identified approximately 200 unique oligosaccharides structures varying from 3 to 22 sugars. The increasing complexity of oligosaccharides follows the general pattern of mammalian evolution though the concentration and diversity of these structures in homo sapiens are strikingly. There is also diversity among human mothers in oligosaccharides. Milks from randomly selected mothers contain as few as 23 and as many as 130 different oligosaccharides. The functional implications of this diversity are not known. Despite the role of milk to serve as a sole nutrient source for mammalian infants, the oligosaccharides in milk are not digestible by human infants. This apparent paradox raises questions about the functions of these oligosaccharides and how their diverse molecular structures affect their functions. The nutritional function most attributed to milk oligosaccharides is to serve as prebiotics - a form of indigestible carbohydrate that is selectively fermented by desirable gut microflora. This function was tested by purifying human milk oligosaccharides and providing these as the sole carbon source to various intestinal bacteria. Indeed, the selectively of providing the complex mixture of oligosaccharides pooled from human milk samples is remarkable. Among a variety of Bifidobacteria tested only Bifidobacteria longum biovar infantis was able to grow extensively on human milk oligosaccharides as sole carbon source. The genomic sequence of this strain revealed approximately 700 genes that are unique to infantis, including a variety of co-regulated glycosidases, relative to other Bifidobacteria, implying a co-evolution of human milk oligosaccharides and the genetic capability of select intestinal bacteria to utilize them. The goal of ongoing research is to assign specific functions to the combined oligosaccharide-bacteria-host interactions that emerged from this evolutionary pressure.
\end{abstract}




\section{Milk and Lactation: Genomics as a Toolset to Reveal Unknown Biological Values of Milk Components}

Milk is the only biomaterial that evolved to nourish growing mammals. Survival of mammalian offspring consuming milk as their sole food exerted a strong Darwinian selective pressure on the biochemical and genetic evolution of the lactation process, leading to the appearance of components that promote health and survival. This evolutionary pressure led to the proteins, peptides, complex lipids and oligosaccharides in higher order structures coming together as a complex, multi-component, yet highly organized food - milk. Although most research has focused on the essential nutrients present in milk, interestingly these are typically as essential to the mother as to the infant. Milk research has thus principally revealed the quantities, forms and bioavailability of the essential nutrients for infants. Nonetheless, in addition to the essential nutrients, the evolution of lactation led to a myriad of nonessential factors. Research to date has recognized that these nonessential compounds, structures and configurations act as growth factors, toxin-binding factors, antimicrobial peptides, prebiotics and immune regulatory factors within the mammalian intestine. Importantly, these trophic macromolecules deliver nutritional functions that, though not essential, provide biological advantages within the intestine and throughout the body that contribute to neonatal mammalian survival. The tools of genomics are beginning to provide the means to apply innovative strategies to accelerate our understanding of the functionality of the subsets of genes that are the products of evolutionary pressure on lactation - those expressed in milk [1,2].

\section{The Biology of Lactation}

Mammary epithelial cell biology research has described the various stages of lactation, the physiology of the mammary gland, the physiology and supporting role of non-epithelial tissues, the energetics of lactation, the regulation of genes and proteins expressed during lactation, and the biochemical processes that occur within lactating mammary epithelial cells and the mammary gland. Unfortunately, to date, little research on lactation biology has focused on the primary underlying driving force for this biological process: the nutrition of the infant.

Milk composition, mammary function and the genes associated with lactation vary across mammalian species. These variations in the composition of milk and in the functions of milk across species point toward the adaptation of milk compositions to the environmental niches, reproductive strategies, and nutrients and growth requirements of different mammalian infants. Thus, the diversity of molecules and structures in different milks reflects the diverse functions of milks emerging through mammalian evolution and the 
mother-infant pair as an intense Darwinian engine. Integrating the various cellular processes of lactation with the nutritional functions of the nonessential molecules and structures produced by lactation will need both the tools of genomics and systems biology and the ingenuity of researchers. Essential nutrient annotation is relatively straightforward, an essential nutrient eliminated from the diet of an infant will produce a deficiency symptom every time, in every infant. However, nonessential components, structures and complexes are presumably beneficial only in a specific situation. Defining the situation is therefore necessary to defining the benefit itself. The development of the mammary gland throughout evolution illustrates the progression and context of the mammary gland to success of the offspring [3].

\section{Infant Nourishment}

Nourishing the mammalian neonate is the most obvious role of milk, and the success of mammals attests to the values of milk as an initial food source for the young of these species. The demands on milk as a sole source of nutrition are remarkable. All of the essential macronutrients, water, vitamins, minerals, amino acids and fatty acids, plus the basic structural and energetic intermediates needed to sustain life, must be delivered to the neonate in a highly absorbable form that is appropriate to the species and the stage of development - all at minimal energy cost to the mother. Lactation research has illuminated many of the biological processes needed to mobilize the essential biomolecules from maternal stores and to convert them into dispersed, transportable and bioavailable structures in milk.

Milk also provides myriad benefits to the growth, development and healthsupporting processes of the young and mothers beyond the essential nutrients. The nonessential components of milk are not understood as well as those of essential nutrients, but research is now beginning to focus on their roles in the wellbeing of neonates. The research strategies needed to discover these properties are different from those used to discover the properties and roles of essential nutrients. Essential nutrients can be studied with relative ease because their elimination from the diet of animals leads to overt signs of deficiency in each individual. Nonessential nutrients and their functions, however, are only valuable within a particular context, thus investigations of benefits of nonessential nutrients must first recognize the context in which they are valuable.

\section{Functions of Milk}

The evolutionary origins of milk proteins and mammary regulation define the key functions of milk and the mammary gland. The evolution of the mammary gland likely involved adaptive recruitment of existing precursor genes 
through alteration of regulatory sequences to allow expression in primitive mammary glands and, duplication and mutation of structural sequences to acquire new functions from preexisting primitive proteins. The earliest mammary function after provision of nutrition was possibly the passing of protective advantages on to offspring by immunoglobulins, thus aiding selection for survival. This paved the way for the elaboration of myriad protective functions that we are only now beginning to appreciate.

The functions of milk generally can be considered supportive of both mammalian mothers and infants through several mechanisms. Milk provides nourishment of infant offspring; disease defense for the infant; disease defense for the mother; regulation or stimulation of infant development, growth or function; regulation or stimulation of maternal mammary tissue development, growth or function; inoculation, colonization, nourishment, regulation and elimination of infant microflora; and inoculation, colonization, nourishment, regulation and elimination of maternal mammary microflora.

\section{Milk Oligosaccharides}

Given the evolution of milk as a product of epithelial secretions nourishing mammalian offspring, the presence of non-digestible oligosaccharides would appear to be paradoxical. The question, why would milk contain indigestible material, has challenged scientists studying milk for decades. The presence and particularly the remarkable abundance of oligosaccharides in human milk as the third largest solid component have led investigators to propose biological, physiological and protective functions to these molecules [4-6]. Certainly, the number and structural diversity of these molecules would allow more than one function. However, to date, the detailed structural basis of these myriad functions is not yet understood. Recently, human milk oligosaccharides (HMOs) have been demonstrated to selectively nourish the growth of highly specific strains of bifidobacteria thus establishing the means to guide the development of a unique gut microbiota in infants fed breast milk [7-9]. Certain oligosaccharides derived from the mammalian epithelial cells of the mother also share common epitopes on the infant's intestinal epithelia known to be receptors for pathogens. The presence of such structures in milk have been hypothesized to have evolved to provide a direct defensive strategy acting as decoys to prevent binding of pathogens to epithelial cells, thereby protecting infants from diseases [10].

Consistent with the potential for multiple nutritional and biological functions, human milk is comprised of a complex mixture of oligosaccharides that differ in size, charge and abundance [11]. HMOs are composed of both neutral and anionic species with building blocks of 5 monosaccharides: D-glucose, Dgalactose, $\mathrm{N}$-acetylglucosamine, L-fucose, and $\mathrm{N}$-acetylneuraminic acid. The basic structure of HMOs include a lactose core at the reducing end and are elongated by $\mathrm{N}$-acetyllactosamine units, with greater structural diversity pro- 
vided by extensive fucosylation and/or sialylation wherein fucose and sialic acid residues are added at the terminal positions [10]. The ability to understand the diversity of biological functions of HMOs has been hindered to date in part because of the lack of detailed structural knowledge of the overall complexity of HMOs in breast milk. At present, about 200 molecular species have been identified in a pooled human milk sample consisting of mostly neutral and fucosylated oligosaccharides [12].

The analytical methods that are capable of separating and characterizing the various sugar compositions and structures of oligosaccharides in human milk include high-performance liquid chromatography (HPLC), high $\mathrm{pH}$ anion exchange chromatography (HPAEC), capillary electrophoresis and various mass spectrometry platforms (MS) [13-21]. These methods as currently used are technically cumbersome, incapable of producing large quantities of highly purified isolated molecules and, as a result, there is little information on many of the basic biological properties of this class of molecule. Even with respect to the variation in abundances and structures across the human condition, little information has been developed. As a result the variation in nourishment that is likely to occur between different infants in different breastfeeding situations is also lacking. Combining the lack of basic information on the diversity of HMOs between different lactating humans, on changes in oligosaccharide compositions and abundances during the course of lactation and on the role of genetic, dietary and physiological determinants on the structures and abundances of HMOs it is difficult to predict at present to what extend variations in health outcomes of different breastfed infants is due to variation in the oligosaccharides delivered in their milk.

To establish the various functions associated with the diverse HMO structures the details of variations in compositions and abundances of oligosaccharides among humans and during lactation need to be measured. Characterization of HMO has been accomplished using HPAEC and HPLC in combination with derivatization techniques [4, 21-24]. The identification of HMOs was based on the retention time of commercially available milk oligosaccharide standards and their quantification was relative to the amount of standards. In one study, a decrease in the total concentration of oligosaccharides was observed from the first weeks postpartum to about half the concentration after one year. In the same report, the absolute and relative concentrations of HMOs between individual donors and at different stages of lactation varied significantly [22]. Asakuma et al. [24] analyzed the level of several neutral oligosaccharides in human milk colostrums for 3 consecutive days from 12 Japanese women. The concentrations of 2 '-fucosylactose and lactodifucotetraose on day 1 were found to be substantially higher than those on days 2 and 3 . On the other hand, the lacto- $N$-tetraose concentration increased from days 1 to 3 . These data are compelling that variation in the structures and abundances of the various oligosaccharides in human milk are variable and it now becomes of considerable scientific and practical interest to understand the regulatory basis of 
these variations (genetics, diet, physiological state of the mother/infant, pathological state of the mother/infant, etc.).

The arrival of HPLC-Chip TOF/MS technology provides the analytical means to take a new strategy to routinely profile oligosaccharides in human milk [9, 11, 12]. This analytical technique employs an integrated microfluidic chip coupled with a high mass accuracy time-of-flight mass analyzer. Using this analytical platform nearly daily profiles of oligosaccharides in human milk samples were determined for different individual human donors (fig. 1). The levels of milk oligosaccharides and their heterogeneity were investigated both within the individual donor and among multiple donors at different stages of lactation. This approach is designed to provide basic knowledge on HMOs in normal humans as the key compositional basis to understanding the relationship between the levels of milk oligosaccharides and the specific functions these biomolecules contribute to maternal and infant health and development.

\section{Analysis of Milk Oligosaccharides}

Milk is a highly complex mixture of biomolecules with overlapping physical, chemical and biological properties. To study each class of these molecules at present it is necessary to isolate them in preparative scale. The initial steps of oligosaccharide chemical, structural and functional analysis used physical methods to separate oligosaccharides from the balance of milk components. Thus cells, lipids and proteins were separated from milk serum [8, 11]. In these laboratory scale studies, a combination of the centrifugation and liquid/liquid extraction was effective in removing lipids, proteins, and small molecules and concentrate oligosaccharides from single milk samples or various pooled milks. The oligosaccharides were desalted and concentrated using GCC-SPE to purify and partially fractionate oligosaccharides. Nonporous graphitized carbon cartridge (GCC; a material similar to PGC; Supelco, State College, Pa., USA) proved to be very effective in removing salts, monosaccharides, detergents (SDS and Triton X-100), proteins (including enzymes), and reagents for the release of oligosaccharides from glycoconjugates (such as hydrazine and sodium borohydride).

Once isolated, various analytical goals were established for the oligosaccharide research. The determination of the precise structural assignments for all HMOs was the first priority. Once in place, however, it was important to develop high throughput methods to analyze large numbers of samples to determine the distributions, concentrations and variations in biological samples from human milk to microbial fermentation studies. Finally, highly quantitative methods were necessary to establish which oligosaccharides were consumed by which bacteria during fermentation studies. This required the development of isotopic enrichment protocols to quantitatively compare entire mixtures of oligosaccharides before and after particular treatments. 


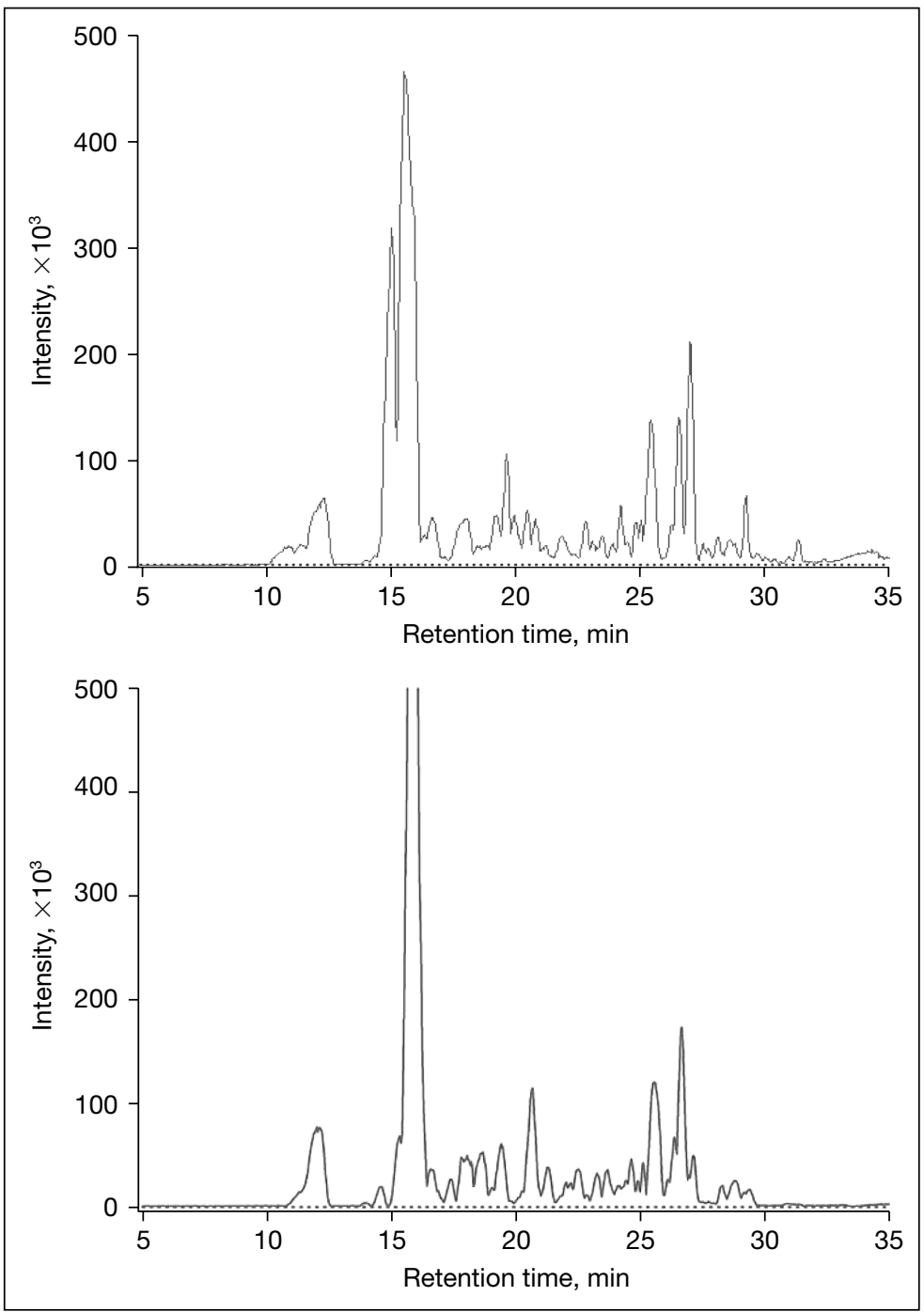

Fig. 1. Comparison of oligosaccharide profiles of breast milk from two mothers on day 6 of lactation. Oligosaccharides analyzed by the Agilent nano-LC chip TOF system.

For structural analyses, the strategy to annotate the human milk glycome was to perform tandem mass spectrometry, specifically infrared multiphoton dissociation, to obtain the sequence and connectivity of each residue and the position of the fucoses and the sialic acids and employ exoglycosidases to differentiate between isomers and determine ambiguous structures [12]. 
For routine analyses of oligosaccharide, samples in high throughput were analyzed using a microfluidic HPLC-Chip/MS technology developed with the active collaboration of Agilent Terchnologies Inc. [11]. The microfluidic HPLC-Chip was made using laser-ablated and laminated biocompatible polyimide film. The chip consists of an integrated sample loading structure, a packed LC separation column, and nanoelectrospray tip. It is hydraulically interfaced with LC pumps and an autosampler through a face-seal rotary valve. A nanoliter pump was used to deliver an LC gradient at $300 \mathrm{nl} / \mathrm{min}$. The chip was interfaced to an o-TOF/MS for online nanoESI. Both the on-chip enrichment column and on-chip LC separation column were packed with porous graphitized carbon media.

For accurate quantitation of oligosaccharides by matrix-assisted laser desorption/ionization-Fourier transform ion cyclotron resonance mass spectrometry (MALDI-FTICR MS), a method using an internal deuterium-labeled standard was developed. In summary, oligosaccharides recovered from the microbial supernatants $(100 \mu \mathrm{l})$ were reduced by adding $100 \mu \mathrm{l}$ of $2.0 \mathrm{~m}$ sodium borohydride or sodium borodeuteride. Isotopic abundance ratios provided a highly accurate and detailed determination of the precise molecular differences between control and treated mixtures [9].

\section{Infant Microflora}

At birth, developing a healthy, protective and metabolically active gut microflora consisting of a wide range of bacteria in various ecological niches along the intestine (termed the infant's microbiome) is considered to be important to the acute health of the infant and the maturation of its intestinal system [25, 26]. Although there are some suggestions that infants are specifically exposed to bacteria prior to birth [27], mammalian infants are generally believed to be born with an intestine that is ostensibly sterile and is hence immediately exposed to various ingested bacteria [28]. Given the likelihood of the infant being challenged by (if not actually perfused in) a large number of aggressive pathogens, and considering the naïve status of both the innate and adaptive immune system of infants, the conditions that lead to and support the bacteria that colonize the infant's intestine have likely been under a very strong Darwinian pressure for survival throughout mammalian and particularly primate evolution. Albeit crude measures of this process have catalogued that breastfed infants are distinctive from adults and non-breastfed infants with an unusual abundance of bacteria generally characterized as bifidobacteria [7]. How Bifidobacteria establish such predominant numbers in breastfed infants and whether their abundance and/or growth properties provide distinct protection to the infant is the subject of intensive ongoing investigations [8, 29]. Several molecules have been proposed as candidates for the putative bifidobacterial growth factors including antimicrobial peptides, 
antibodies and milk oligosaccharides [5, 30]. Hence it is of considerable interest to know precisely how different bacteria are established in the gut of the newborn infant and in particular to understand what in breast milk leads to the unusual accumulation and persistence of the protective microbiome of breastfed infants. Therefore, a research strategy was developed to ascertain whether the oligosaccharides in human milk when isolated would provide a highly selective growth response in different species of intestinal bacteria.

\section{Selective Microbial Fermentation of Milk Oligosaccharides}

The functions of oligosaccharides in stimulating the growth of beneficial bacteria remain poorly understood. If oligosaccharides in milk are to be net beneficial by virtue of their selective stimulation of uniquely protective bacteria, the mechanisms behind this selectivity must be understood. Oligosaccharides purified from human milk were provided as the sole carbon source in a microbial growth assay inoculated with various bacteria. The bioselectivity of these oligosaccharides for specific bacterial growth are remarkable. Growth curves against time for 6 strains of Bifidobacteria are shown in figure 2. While all strains of Bifidobacteria grew well on lactose and several grew successfully on inulin, only Bifidobacteria longum biovar infantis grew successfully on HMOs as the sole carbon source supporting their growth.

\section{Oligosaccharide Specificity by Different Bacteria}

To determine the precise nature of the oligosaccharides that are consumed by different bacteria, the deuterium isotope enrichment technique was used to measure the difference in abundance of each of the different oligosaccharides before and after microbial fermentation. The flow sheet describing this technique is shown in figure 3a, and typical results in figure 3b. By incubating various bacteria with the milk oligosaccharide mixture and quantifying each of the respective mass-based structures, it is possible to build a map of the grazing strategy of different bacteria [31]. Using this approach $B$. longum bv. longum, $B$. longum bv. infantis, and $B$. breve, used as example bifidobacterial species isolated from the gut in infant and adults, were profiled for their selectivity in fucosylated and neutral HMO consumption oligosaccharides. $B$. infantis preferably consumed oligosaccharides with a degree of polymerization of $\leq 7$ (m/z 1389 and below). These are quantitatively the most abundant oligosaccharides in pooled human milk. These data further suggest a direct relationship between what the mammary gland is producing and what the $B$. infantis strain consumes.

The implications of these data are clear. With infants consuming breast milk and digesting and absorbing all biomolecules possible, the abundance of 


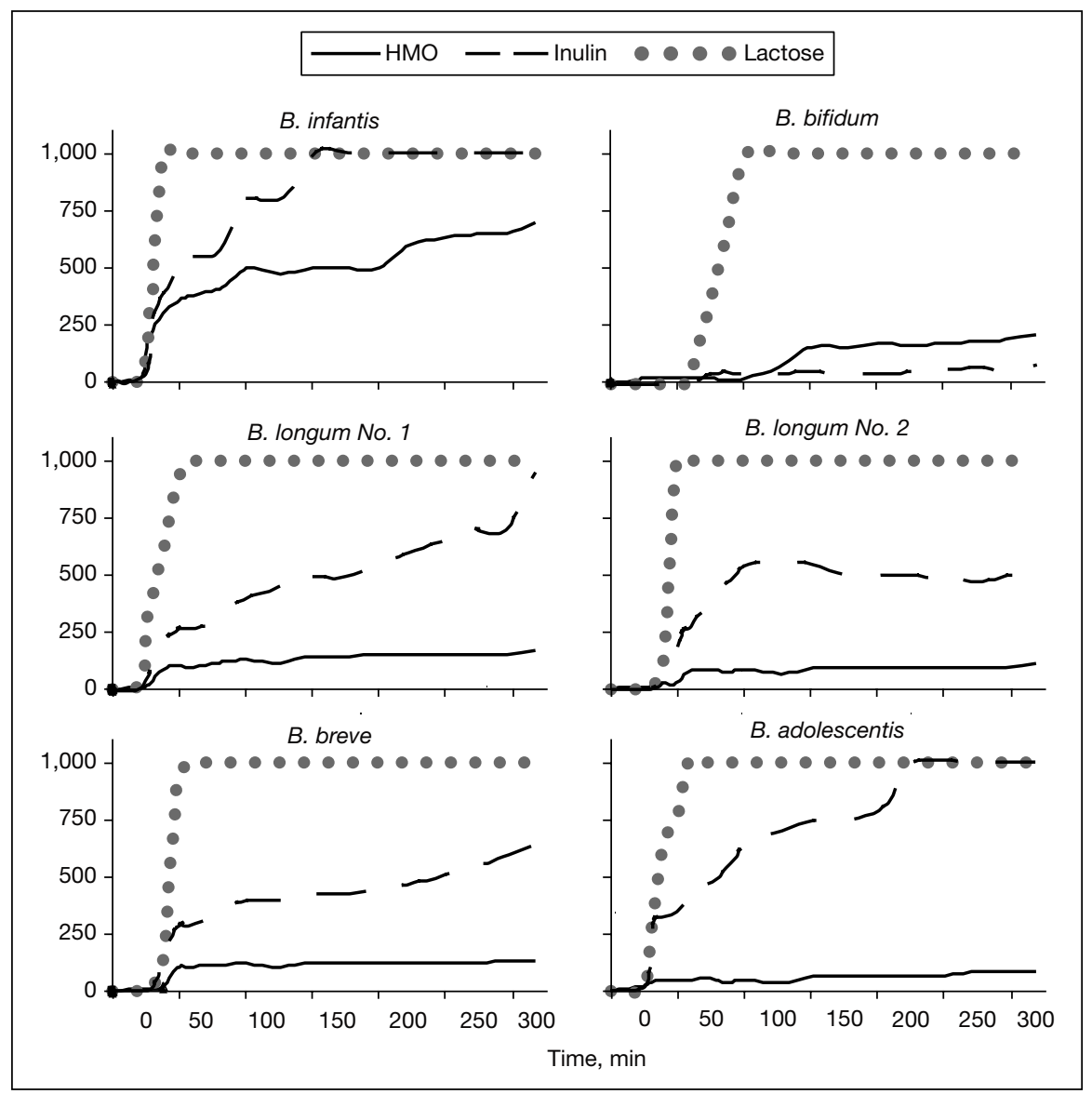

Fig. 2. Growth curves for different strains of bacteria using lactose, inulin and human milk oligosaccharides (HMO) as their sole carbon source. Modified from Ward et al. [8].

the milk oligosaccharides combined with the lack of enzymatic capabilities by the infant to digest them, the major biomolecule class reaching the infant's lower intestine will be the complex oligosaccharides. All bacteria that are attempting to colonize the infant's intestine will be forced to compete based on these oligosaccharides as their major carbon source. This places a prohibitive premium on the genetic capabilities of different bacteria to consume oligosaccharides. It is now the focused objective of this research to identify various strains of bacteria capable of consuming HMOs and to characterize 


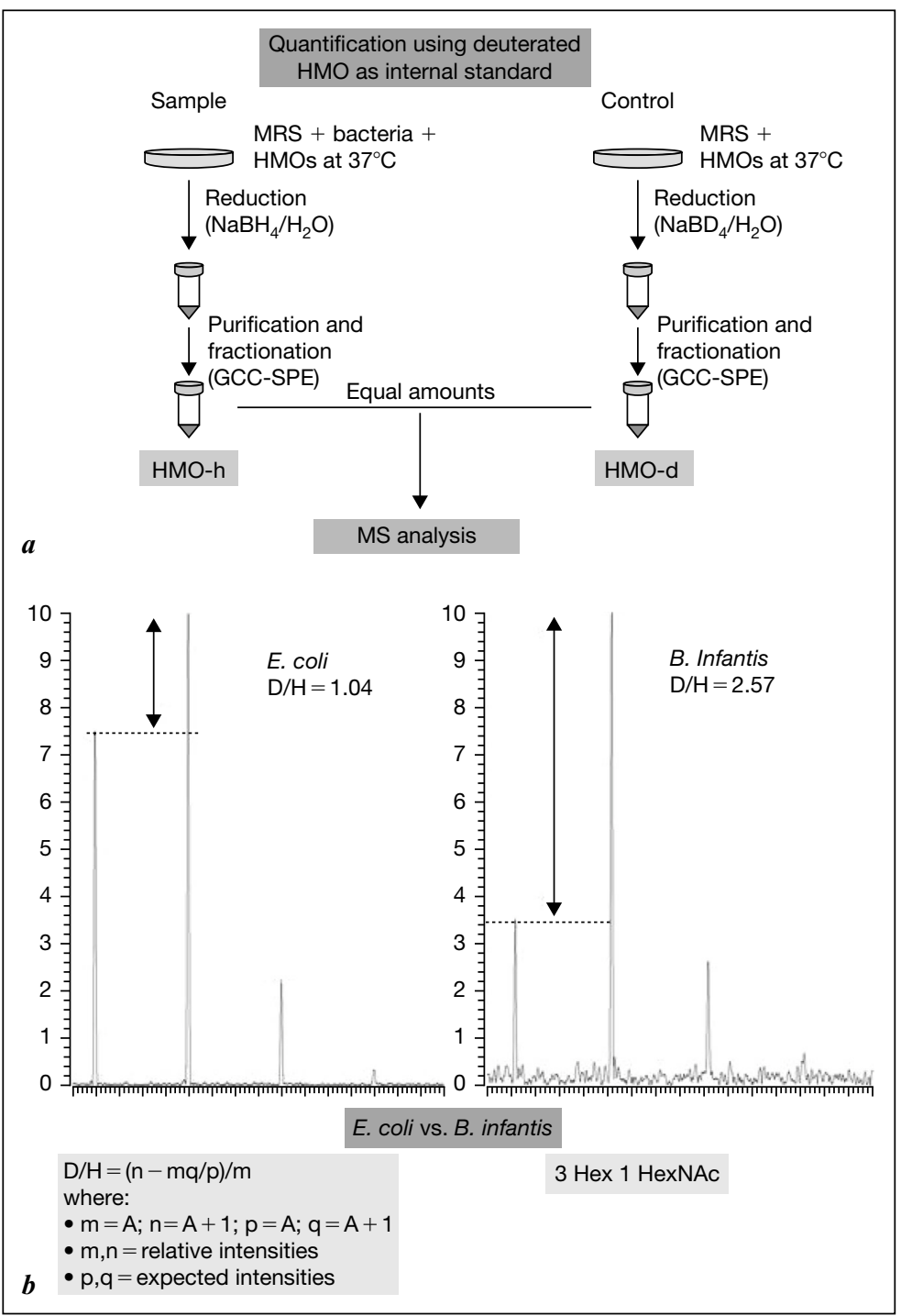

Fig. 3. a Flow sheet of the method of glycoprofiling using isotope enrichment and mass ratio quantitation. $b$ Typical data comparing $E$. coli and B. infantis consumption of specific oligosaccharides.

the genomes of these organisms with sufficient detail to understand the basis of their unique ecological niche (breastfed infants) and the Darwinian advantage that this remarkable product of co-evolution provides to the breastfed infant. 


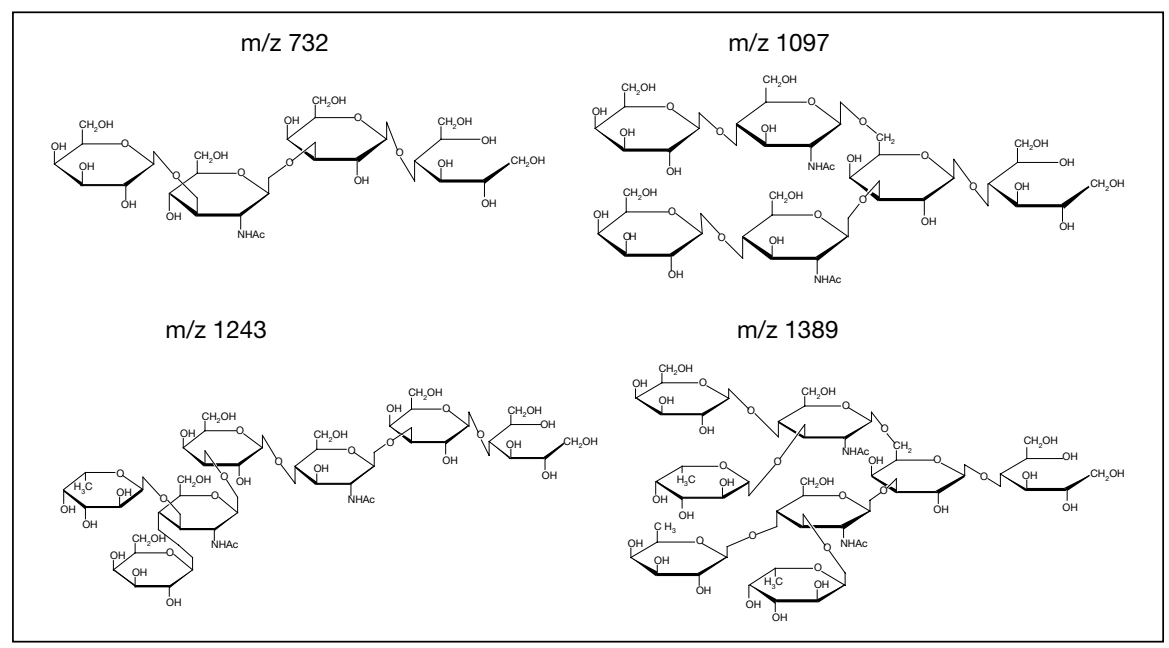

Fig. 4. Structures of human milk oligosaccharides consumed by Bifidobacteria infantis.

\section{Personalization of Infants}

It is intriguing to speculate on the role of the diverse oligosaccharide products found in human breast milk. Results to date demonstrate clearly that samples of breast milk from different mothers exhibit different concentrations, structures and lactational variation in their oligosaccharides. Do these variations provide infants with an unusual advantage? Do variations in the physiology of the mother or the infant trigger these variations? Would infants in particular environments, growth stages or physiological states take advantage of selective variations in the amounts of oligosaccharides? These are questions that must await future research to be resolved. Nonetheless, the intimate relationship between breast milk, infants and their resident microflora argues compellingly for active research in this area. It should be undertaken quickly to determine how oligosaccharide levels vary in different humans. Once accurate methods are in routine practice around the world, it will be possible to map the composition of HMOs with various health outcomes from diarrhea to allergy. 


\section{References}

1 German JB, Dillard CJ, Ward RE: Bioactive components in milk. Curr Opin Clin Nutr Metab Care 2002;5:653-658.

-2 German JB, Schanbacher FL, Lönnerdal B, et al: International milk genomics consortium. Trends Food Science \& Technology 2005; vol 17(12):656-661.

-3 Schanbacher FL, Talhouk RS, Murry FA: Biology and origin of bioactive peptides in milk. Livestock Production Science 1997; vol 50(1-2):105-123.

-4 Coppa GV, Gabrielli O, Pierani P, et al: Changes in carbohydrate composition in human milk over 4 months of lactation. Pediatrics 1993;91:637-641.

-5 Kunz C, Rudloff S, Baier W, et al: Oligosaccharides in human milk: structural, functional, and metabolic aspects. Annu Rev Nutr 2000;20:699-722.

6 Bode L: Recent advances on structure, metabolism, and function of human milk oligosaccharides. J Nutr 2006;136:2127-2130.

-7 Harmsen HJM, Wildeboer-Veloo ACM, Raangs GC, et al: Analysis of intestinal flora development in breast-fed and formula-fed infants by using molecular identification and detection methods. J Pediatr Gastroenterol Nutr 2000;30:61-67.

8 Ward RE, Niñonuevo M, Mills DA, et al: In vitro fermentation of breast milk oligosaccharides by Bifidobacterium infantis and Lactobacillus gasseri. Appl Environ Microbiol 2006;72: 4497-4499.

-9 Niñonuevo MR, Ward RE, LoCascio RG, et al: Methods for the quantitation of human milk oligosaccharides in bacterial fermentation by mass spectrometry. Anal Biochem 2007;361: $15-23$.

10 Newburg DS, Ruiz-Palacios GM, Morrow AL: Human milk glycans protect infants against enteric pathogens. Annu Rev Nutr 2005;25:37-58.

-11 Niñonuevo M, An H, Yin H, et al: Nanoliquid chromatography-mass spectrometry of oligosaccharides employing graphitized carbon chromatography on microchip with a high-accuracy mass analyzer. Electrophoresis 2005;26:3641-3649.

12 Niñonuevo MR, Park Y, Yin H, et al: A strategy for annotating the human milk glycome. J Agric Food Chem 2006;54:7471-7480.

13 Thurl S, Muller-Werner B, Sawatzki G: Quantification of individual oligosaccharide compounds from human milk using high-pH anion-exchange chromatography. Anal Biochem 1996;235:202-206.

14 Chaturvedi P, Warren CD, Ruiz-Palacios GM, et al: Milk oligosaccharide profiles by reversedphase HPLC of their perbenzoylated derivatives. Anal Biochem 1997;251:89-97.

15 Charlwood J, Tolson D, Dwek M, Camilleri P: A detailed analysis of neutral and acidic carbohydrates in human milk. Anal Biochem 1999;273:261-277.

16 Nakhla T, Fu DT, Zopf D, et al: Neutral oligosaccharide content of preterm human milk. Br J Nutr 1999;82:361-367.

17 Shen ZJ, Warren CD, Newburg DS: High-performance capillary electrophoresis of sialylated oligosaccharides of human milk. Anal Biochem 2000;279:37-45.

18 Suzuki M, Suzuki A: Structural characterization of fucose-containing oligosaccharides by high-performance liquid chromatography and matrix-assisted laser desorption/ionization time-of-flight mass spectrometry. Biol Chem 2001;382:251-257.

19 Pfenninger A, Karas M, Finke B, Stahl B: Structural analysis of underivatized neutral human milk oligosaccharides in the negative ion mode by nano-electrospray MS(n) (part 1:methodology). J Am Soc Mass Spectrom 2002;13:1331-1340.

-20 Pfenninger A, Karas M, Finke B, Stahl B: Structural analysis of underivatized neutral human milk oligosaccharides in the negative ion mode by nano-electrospray MS(n) (part 2:application to isomeric mixtures). J Am Soc Mass Spectrom 2002;13:1341-1348.

-21 Sumiyoshi W, Urashima T, Nakamura T, et al: Determination of each neutral oligosaccharide in the milk of Japanese women during the course of lactation. Br J Nutr 2003;89:61-69.

-22 Chaturvedi P, Warren CD, Altaye M, et al: Fucosylated human milk oligosaccharides vary between individuals and over the course of lactation. Glycobiology 2001;11:365-372.

23 Musumeci M, Simpore J, D'Agata A, et al: Oligosaccharides in colostrum of Italian and Burkinabe women. J Pediatr Gastroenterol Nutr 2006;43:372-378.

24 Asakuma S, Urashima T, Akahori M, et al: Variation of major neutral oligosaccharides levels in human colostrum. Eur J Clin Nutr 2007 [Epub ahead of print]. 
25 Kelly D, Conway S, Aminov R: Commensal gut bacteria: mechanisms of immune modulation. Trends Immunol 2005;26:326-333.

26 Forchielli ML, Walker MA: The role of gut-associated lymphoid tissues and mucosal defence. Br J Nutr 2005;93(suppl 1):S41-S48.

-27 Perez PF, Doré J, Leclerc M, et al: Bacterial imprinting of the neonatal immune system: lessons from maternal cells? Pediatrics 2007;119:e724-e732.

28 Fanaro S, Chierici R, Guerrini P, Vigi V: Intestinal microflora in early infancy: composition and development. Acta Paediatr Suppl 2003;91:48-55.

29 Palmer C, Bik EM, Digiulio DB, et al: Development of the human infant intestinal microbiota. PLoS Biol 2007;5:e177.

30 Gyorgy P, Norris RF, Rose CS: Bifidus factor. I. A variant of Lactobacillus bifidus requiring a special growth factor. Arch Biochem Biophys 1954;48:193-201.

-31 LoCascio RG, Ninonuevo MR, Freeman SL, et al: Glycoprofiling of bifidobacterial consumption of human milk oligosaccharides demonstrates strain specific, preferential consumption of small chain glycans secreted in early human lactation. J Agric Food Chem 2007;55: 8914-8919.

\section{Discussion}

Dr. Isolauri: You verbalized the historical view that human milk oligosaccharides (HMOs) are good and bifidobacteria are good, and thus we should increase bifidobacteria biota as if the bifidobacterium level were a health benefit per se. We have shown for instance that allergic infants have different bifidobacteria than healthy infants. So it makes a lot of sense that also HMOs have specific effects on different bifidobacteria.

Dr. German: One of the things that has actually been very intriguing is that we had no idea that this was going to be the way things worked out. We were very surprised about the differences in bifidobacteria and frankly the opportunity to now begin to assign these functions. We have started working with a company to deliver DNA chips so we can provide the means, for your program for example, to analyze quantitatively all the bifidobacteria in a very specific way, not by the technique of culture but by identifying each strain, which we think is actually going to be necessary now that it is clear that they are so different.

Dr. Walker: Does the quantitative composition of oligosaccharides substantially change with time?

Dr. German: As mothers, humans are about the most persistent oligosaccharide producers that we have found. Although primates for the most part tend to persist in their oligosaccharide support, rhesus monkeys, for example, are relatively similar to humans, but humans hold onto that production capability. But it is still too early to know exactly what the basis is. At the present time there is no evidence that there is a rapid decline in a specific subset or a specific group, they persist for quite a long time much of the way through lactation.

Dr. Walker: It is my understanding that in the first short period of postpartum life bifidobacteria are very important players but then as other organisms expand they play a lesser role. Does that have to do with other substrates that are available to stimulate colonizing bacteria?

Dr. German: From an ecological point of view it becomes very interesting because basically as long as the oligosaccharides in human breast milk are those things that are coming along, initially the bifidobacteria will in essence proliferate. The strategy of the mother recruiting an entire life form to babysit her infant is ingenious. But you can imagine that the bacteria that are going to begin to succeed in that environment will be the ones that can take that which the bifidobacteria are throwing away, and we see evidence of that already, such as your data that the gut begins itself to nourish. As the 
gut itself begins to mature successfully, it takes over a very important feeding role, and diversity begins to establish. It could be that not only are the initial bifidobacteria directed through oligosaccharides, but also a subsequent proliferation of species. Although this is a very recent elaboration in evolution, the long oligosaccharides seem to be the ones in similar studies showing binding. How these will in essence be able to provide surrogate binding sites for other bacteria even viruses is not yet known, so the ways with which the milk is regulating the intestinal ecosystem is quite complex. The fact that we are so unique relative to other primates suggests that there have been some things that humans have done in their recent history that unusually threatened infants from a pathogenic perspective, which again may say that unless there is a very threatening pathogenic environment, benefit won't necessarily be seen, and that may be why infant formula that does not contain these things hasn't shown any substantial difference in outcome. We haven't put infants in the sorts of pathogenic environments. It is possible that relatively similar primates don't have the amounts because they are relatively solitary and their infants are not exposed to the sorts of unusual clustered living that humans are.

Dr. Walker: Because oligosaccharides represent such a high percentage of the total content of breast milk, I always think that they are clonically fermented and then short-chain fatty acids were being taken up as an energy source. But from what you said, it seems that there is not so much fermentation going on. What is their use in addition to that; have you thought about that evolutionarily?

Dr. German: We have, and to a certain extent we have to start back at square one now that these molecules can be analyzed in high throughput, and especially to measure the entire ensemble, expose them to bacteria, viruses, etc., and then see what oligosaccharides are selectively taken up. Then we can identify and begin to functionally annotate those molecules. It is going to be a fascinating area of nutrition.

Dr. Bier: The classification of bacteria was at least historically based on relatively crude phenotypic types of things, and here you have given us one, I think these have an $n$ of 1 genome. How stable is this genome in that species across the human population; what are the differences between those adult and infant genomes, and do we know, for example, in the gut ecology of the infant that the functions that aren't present in one bacteria aren't replaced by something else in the ecological system? It seems that we should at least have an $n$ of 2 or 200 , or something.

Dr. German: With the Joint Genome Institute (JGI) we have just finished sequencing 8 more. We have picked up some from sources in India, Africa and a couple in the United States, so even within Bifidobacterium infantis there are interesting differences within the genome. So there is a certain amount of diversity there, and it is certainly going to be an interesting area. There are other bacteria that look as though they will be able to grow but not as successfully and perhaps not as competitively. But interestingly it may mean that if you are not inoculated with the $B$. infantis you might begin to get some unusual bacteria which may be one of things that we see from cesarean section infants, for example. We are sequencing as fast as the JGI will give us capacity on sequencers, and thankfully we are getting better. We also want to be able to build the tools to be able to take this to human infant samples and begin to document that they are there in numbers and abundance. Also, where they are in human mothers because clearly they are being inoculated. If these bacteria are passed from mother to daughter through generations, a cesarean section in a sterile birth and incubator growth, keeping the infant away from these, could actually break that chain, and therefore at least the daughters should be re-inoculated so that they can keep that legacy going.

Dr. Räihä: Do the oligosaccharides cross the placenta? I am asking this because of the difference between a fetus who is developing to term in utero versus a fetus who is born prematurely and then receives quite a lot of oligosaccharides from the mother. 
Dr. German: We are actually now taking the tools to a variety of other biofluids, and certainly the placenta will be studied. At this point we don't know; there are no data, and this must be pursued.

Dr. Gluckman: Do you know anything about maternal health and effects on oligosaccharide profile or maternal nutritional profile? You said each mother produces only roughly 130 over 200 possibilities. What about the 70 that are not produced? Is there a consistent pattern where the high variation is in the long chain?

Dr. German: No, that's interesting, in fact when you say 130 that doesn't mean that all mothers produce 130; there are some mothers who are producing only approximately 35. So there is variation and we don't know the difference. We are now in the process of recruiting samples from all over the world, from India, Gambia, South America, to answer those questions. Because there actually are infants and mothers in much more difficult situations in many respects and greater variation in outcomes, we think we can begin to assign some of those differences to more functional endpoints.

Dr. Gluckman: Is there a core group that is always produced?

Dr. German: The ones I showed are always there. To date we have never found a mother who doesn't produce the core that supports the $B$. infantis; the variation is primarily in the long ones.

Dr. Kunz: There was excellent work from Sweden, the United States, and Finland about 25-30 years ago demonstrating exactly 150 oligosaccharides. Coming back to the placenta, as far as we know these oligosaccharides are produced exclusively in the mammary gland and are based upon lactose. In my opinion there is no reason why they should pass the placenta unless we find other places where they are produced. You mentioned that these oligosaccharides are not digested. That's true but it doesn't mean that they are not absorbed. By using stable isotopes, we have shown in term and preterm infants that a small percentage (1-2\%) are excreted in the urine of these infants [1]. Depending on the blood group and the secretory specificity, if an infant receives per suckling 50-150 mg bactoentitrose, which is the core structure, this must be absorbed and nobody knows exactly why. But this amount circulates in the blood and some will be excreted. This means that we have to investigate the systemic effects of milk oligosaccharides as well. I also agree that in animal species there is only a low number of milk oligosaccharides, basically of sialylated components. Each species, goat milk, sheep milk, gorilla and so on, all has an individual pattern. We don't know yet what this means. You showed 6 or 7 structures which are very specific regarding the functions of the bifidobacteria. The amount of oligosaccharides depends very much on the mother's Lewis specificity and secretory status. Meaning that if samples are analyzed, within a few days the Lewis activity, Lewis A or Lewis B activity or the secretory status, is known. Then there are 3 different patterns: the first group has a large amount of oligosaccharides 120-150; in the second group there are perhaps only 100, and the third group, only $10 \%$ of the population, is non-secretor and Lewis B and Lewis A negative and only has about 50 or 60 . What do these very different patterns of milk oligosaccharides mean?

Dr. German: Actually that was one of the reasons for the underlying enthusiasm for the subject of this meeting and this workshop. There are aspects of diversity within the human condition that when looked at in some initial clinical trials of, for instance, diarrhea outcomes in Mexico City, differences in diarrhea in children can be assigned to secretory status. So we are confident that there is a pot of gold at the end of this but it is going to take a substantial amount of research. What we are going to establish are these encouraging specific bacteria, and then, are they binding to specific pathogens? This is a field that needs very good high throughput tools. It is a very important thing, and while bacterial stimulation has been our focus up until now, I think we are ready to begin to answer that sort of question, but it is going to take large studies. Individualizing outcomes takes a great deal of resources. 
Dr. Corthésy: A very provocative presentation, at least for microbiologists. Since milk contains a high amount of lactose and the gut is lined with highly glycosylated proteins, why would bacteria select HMO to grow?

Dr. German: The initial thought would be that the lactose goes to the infant and the infant digests and absorbs that long before the lower intestinal bacteria so what we are basically likely to see is that the intestine is excluding everything but the oligosaccharides because of the infant's role in competing for digestible and absorbable molecules. The hidden oligosaccharides or next phase of glycobiology actually is in all the glycosylated proteins and lipids and I think that is going to make this area at least as complicated. If you look at the proteins and the extent of diversity of glycosylation of proteins and the fragments you get from proteolysis, one would argue that if a protein was only providing amino acids for nutrition, evolution would not select to decorate it and leave it as amino acids to be rapidly absorbed. If proteins were glycosylated by a sort of a backwards evolutionary logic there is a likely function to that and that means that we are also going to be enriching the infant intestine with specific glycosylated peptides for example, and how the different bacteria then compete for that will be another aspect of the ecology. Unfortunately Carlito Lebrilla and his group are still developing the tools for high throughput glycoproteomics, so by next year we should be able to report on those methods, but it is going to change the story as well. We still don't know what lactose does. I think that lactose may actually participate in this mother-infant conflict. We don't talk about this, but the infant has a 100\% investment in its mother's milk. However, the mother has only a 50\% investment in feeding the infant and so there is a fundamental conflict. The mother wants to wean off that infant and so while stopping breastfeeding is in the interest of the mother, it is not in the interest of the infant. How do you do that? One thought, and it is a very interesting idea, is that lactose is there in part because lactase can then be shut off, and lactose then becomes unpleasant and the breastfeeding experience becomes unpleasant. So looking at evolution and all the aspects of milk, there may be some things in milk that are of benefit to the mother-infant pair that are not actually in the infant's best interest. So when we start looking at the overall picture, it is going to be complicated.

Dr. Berry: As I recall, there are 2 or 3 marine mammals whose breast milk doesn't contain lactose. Have you had a chance to look at some of these more exotic species to see if oligosaccharides are missing?

Dr. German: We haven't been that extensive yet. In terms of our approaches, we have primarily been looking at the primate lineages to see if we could explain when and where they appeared and perhaps then be able to speciate the bacteria associated with that. There are certainly anecdotes of some very interesting animals and what they eat and their oligosaccharides. We have elephant milk because it is supposed to be very rich in milk oligosaccharides as well, but we're early on and in those sorts of analyses.

Dr. Berry: Are you suggesting that lactose is the building block for the oligosaccharides? If there is no lactose, then theoretically oligosaccharides couldn't be made?

Dr. German: There are two questions. One is if lactose can't be made, then the oligosaccharides can't be made, but lactose can actually be made, and just completely decorates all of it. Actually I believe marsupials can produce lactose. So lactose even though we think it is mammalian, it is a little bit earlier than that and defines that decision to become an epithelial secretor of these materials. Interestingly in the early development of kangaroos and similar marsupials, they appear to live basically on lactose and oligosaccharides.

Dr. Netrebenko: In the case of bottle feeding, what kind of oligosaccharides, prebiotics, could be the best source for the growth of $B$. infantis? Are there differences between the protective function of, for example, B. infantis and Bifidobacteria longum? 
Dr. German: To be honest in terms of what you would supply to get the function of the $B$. infantis selection is right now one of the big challenges. What we see from things like inulin, is it supports a pretty discouraging broad range of bacteria and so I am not sure that in the long term we will think of inulin as being an infant-appropriate oligosaccharide source. The great dilemma for any one in the industrialization of this is where you are going to get HMOs. They are unique, and remember if they proliferate in a variety of places then bacteria would have proliferated, those gene sets would have been co-opted presumably by a variety of pathogens and lose selectivity. The heart of glycobiology is this constant competition between the organism decorating itself and pathogens trying to find adherence. Basically when a pathogen finds a way to do that, then disease occurs; the flue epidemics are a classic example where a new viral form is able to bind and get into human cells because of that difference. What we see in the commercial future is first oligosaccharides identical to human milk, but then there is no reason why we couldn't actually enhance this whole dimension to accelerate the evolution of humans to keep ahead of pathogens. The future of this I think is very exciting. Human milk has given us a paradigm but I would not think that industrially we will stop there. One could imagine actually getting much more sophisticated and the future probiotics being very sophisticated and individualized bacteria that are being selected for and evolved industrially to live on oligosaccharides that we are able to make. We will never make them more simple, we will make them more complex.

Dr. Strandvik: The mucous layer in the intestine needs fucose and sialic acid for the synthesis. Might it be that in the newborn and in early life the oligosaccharides are just substrates for the mucus before infants get other feeding?

Dr. German: Absolutely, sialidase may actually be one of the benefits of these bifidobacteria, that they are actually able to take sialic acid off the oligosaccharide and deliver it to the intestinal cells. That is certainly one of the hypotheses we are pursuing, the gene capability is there. I think it is a really good idea and would be one of the possible reasons to explain this co-evolution that bacteria are providing a catalytic activity that in essence doesn't exist in the infant.

Dr. Strandvik: that Would also explain a little about some of the variation because the fucosyltransferases are genetically determined in the human [2]. So that might be one point to explain the variation.

Dr. German: Absolutely and when you think about it from an evolutionary perspective, metabolites can frequently evolve faster, especially in a quantitative sense, than whole gene products. So in essence glycobiology is a great evolutionary machine itself and so you can see a rapid change in oligosaccharide amounts and patterns without massive changes in genes, but in the activities and regulation of the complement of enzymes.

\section{References}

$>1$ Rudloff S, Pohlentz G, Diekmann L, et al: Urinary excretion of lactose and oligosaccharides in preterm infants fed human milk or infant formula. Acta Paediatr 1996;85:598-603.

-2 Henry SM: Molecular diversity in the biosynthesis of GI tract glycoconjugates. A blood-grouprelated chart of microorganism receptors. Transfus Clin Biol 2001;8:226-230. 